Document downloaded from:

http://hdl.handle.net/10251/51772

This paper must be cited as:

Guerrero Cortina, F.; Santonja, F.; Villanueva Micó, RJ. (2011). Analysing the Spanish smoke-free legislation of 2006: A new method to quantify its impact using a dynamic model. International Journal of Drug Policy. 22(4):247-251. doi:10.1016/j.drugpo.2011.05.003.

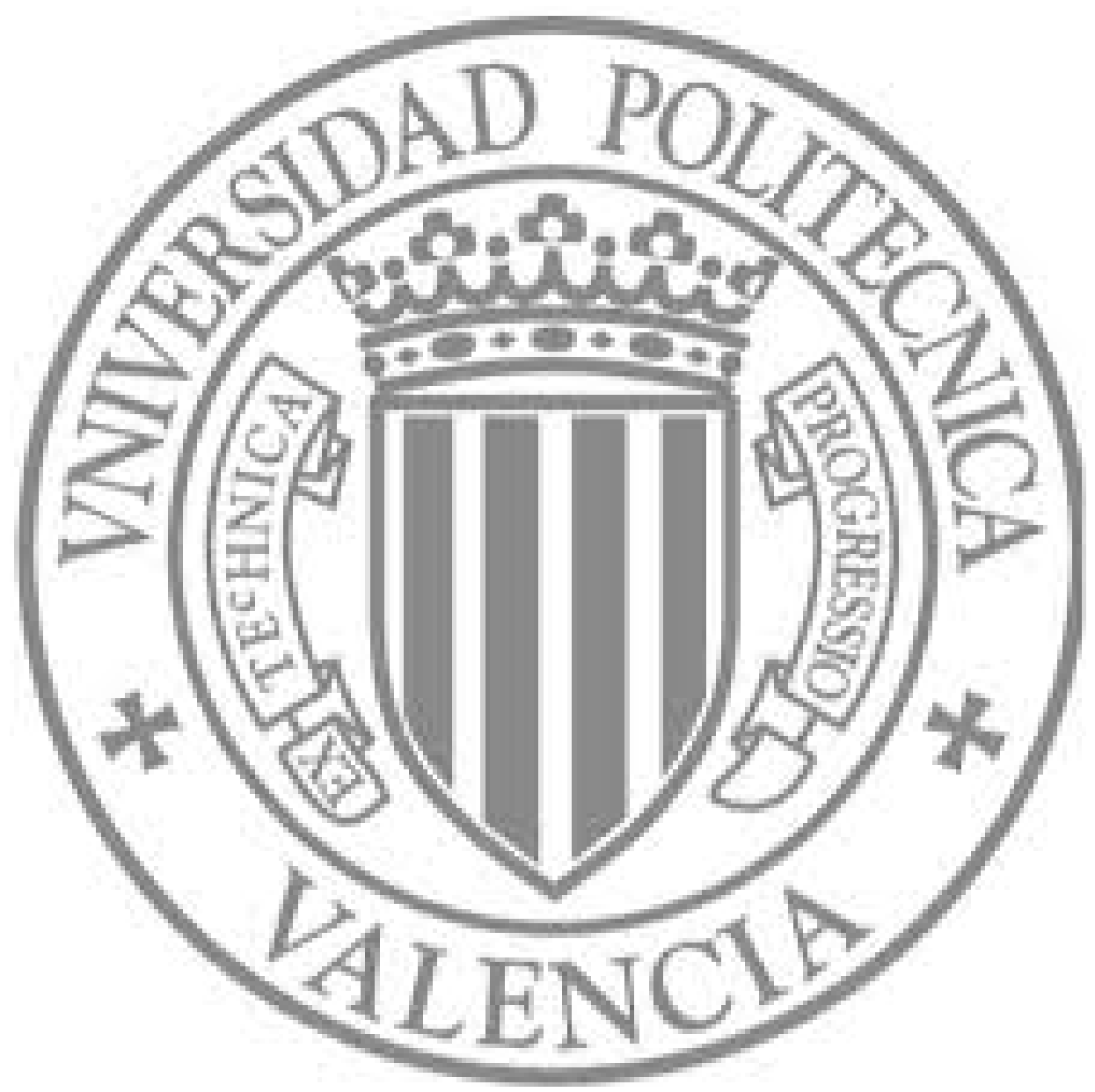

The final publication is available at

http://dx.doi.org/10.1016/j.drugpo.2011.05.003

Copyright Elsevier 
TITLE PAGE

Analysing the Spanish smoke-free legislation of 2006: A new method to quantify its impact using a dynamic model.

Corresponding autor:

Francisco Guerrero.

Dept. Matemática Aplicada. Facultad de Matemáticas. Universidad de Valencia.

Avda. Doctor Moliner, 50. 46100 - Burjasot. Valencia. Spain.

Email: guecor@uv.es

Tel. : +34963543232

Fax: +34 963543922

Co-authors:

Francisco-José Santonja

Dept. Estadística e Investigación Operativa. Universidad de Valencia. Burjasot. Valencia. Spain.

Rafael-Jacinto Villanueva

Instituto de Matemática Multidisciplinar. Universidad Politécnica de Valencia. Valencia. Spain.

Word count: $\underline{2650}$ 


\title{
Analysing the Spanish smoke-free legislation of 2006: A new method to quantify its impact using a dynamic model.
}

Keywords: Tobacco smoking, Simulation model, Health policy evaluation.

\begin{abstract}
Background: There are many models that study different aspects concerning smoking habits: influence of price, tax, relapse time, the effects of prohibition, etc. There are also studies on the effect of the Spanish smoke-free law, but from a statistical point of view, not from a dynamic point of view. We wanted to build a model able to separate the effect of the law from the pre-law evolution of smoking habits.
\end{abstract}

Methods: Using data from the Spanish Ministry of Health and Social Policy, we have developed a dynamic model of tobacco use. The model projects the evolution over time of the number of non smokers, smokers and ex-smokers before 2006. Then, we compare the predictions of the model with real data for the years after the law came into force, 2006 and 2009.

Results: We show that smoke-free law has had a significant impact on different subpopulations. The number of ex-smokers increased significantly in 2006 and maintained the same value in 2009. The number of smokers also decreased significantly in 2006, but in 2009 this returned to its value before the law. Simultaneously, the number of non-smokers decreased in 2009.

Conclusions: When the law came into force (2006), its restriction on smoking in public and work places made many smokers decide to give up smoking. Then the number of smokers decreased, and obviously, the number of ex-smokers, increased. In 2009, the majority of those who succeeded in giving up smoking did not return to the smoking habit. However, the smoke-free law had no effect on new smokers and the number of smokers returned to previous values, whereas the number of nonsmokers decreased. Therefore, we can conclude that, the law had a very positive effect in the first years but this effect dissipated as time was gone on, with the exception that the number of exsmokers, at present, is still higher than before the law. 


\section{INTRODUCTION}

Tobacco smoking is a serious health concern not only from the individual health point of view but also from the public socioeconomic one. In Spain, it is estimated that around 55,000 deaths each year are attributable to smoking (Banegas et al.,2005). Because of this fact, the Spanish Administration introduced a smoke-free legislation in 2006 prohibiting smoking in enclosed public and work places (National Law Number: 28/2005 (Ministry of Health and Social Policy,2005)).

There are some studies on the impact of smoking-free laws in other countries (Erazo et al.,2010; Lock et al.,2010). For instance, (Erazo et al.,2010) analyses air nicotine concentrations before and after the law, using linear regression models on log-transformed nicotine. Additionally, (Lock et al.,2010) analyses smoker's behavior in different ethnic groups using a longitudinal and qualitative panel study of smokers.

The evolution of tobacco use in Spain is shown in Table 1. The data on smoking prevalence are from the 1995-2003 National Health Survey (Ministry of Health and Social Policy,2006). The smoking data are part of a survey which includes data on household characteristics, health and healthcare utilisation. The survey was for the over-16s. Individuals who had not smoked a total amount of 100 cigarettes during their life were considered non-smokers. Normal smokers are anyone who smokes less than 20 cigarettes daily. Excessive smokers are anyone who smokes more than 20 cigarettes daily. Ex-smokers are anyone who has smoked in the past but does not currently smoke.

As proposed in (Christakis \& Fowler,2009; Christakis \& Fowler,2008), tobacco use can be considered as a socially transmitted habit. This leads us to develop an epidemic-type mathematical model (based on a system of differential equations) to study the evolution of tobacco use in Spain as a habit that may be spread by social contagion. In this paper, we present the model and use it to analyse the effect of the smoke-free law which came into force on January 1st, 2006.

This technique has been successfully applied in other socially transmitted habits related to public health such as alcoholism (Santonja et al.,2010) and cocaine consumption (Sanchez et al.,2010).

\section{$<<$ Insert Table 1 $>>$}

In this article, taking into account these data (Table 1) we predict smoking prevalence in the years after the Spanish smoke-free law came into force and compare these predictions (without smoke-free law) with the real smoking rates (with smoke-free law). This allows us to analyse the gap between the predicted and the real prevalence, in order to quantify the effect of the law.

This paper is organized as follows. The model used to simulate the effects of the law under study is presented in the section Methods. Section Results presents the quantification of the effects of this Spanish public policy. The last section is devoted to conclusions.

\section{METHODS}

\section{The model}

In this approach, we take smoking to be a disease that spreads through peer pressure or social contact. The main idea behind this approach is that tobacco use may spread from one person to 
another (Christakis \& Fowler,2009; Christakis \& Fowler,2008). These facts lead us to propose an epidemiological mathematical model to study the evolution of the prevalence of smoking in the Spanish population (16-65 years old).

Taking into account the National Health Survey for Spain (Ministry of Health and Social Policy,2006), Spanish population (16-65 years old) is divided into four subpopulations : N, nonsmokers, $\mathrm{F}$, normal smokers, $\mathrm{F}_{\mathrm{c}}$, excessive smokers and $\mathrm{E}$, ex-smokers.

The transitions between these subpopulations are shown graphically in Figure 1.

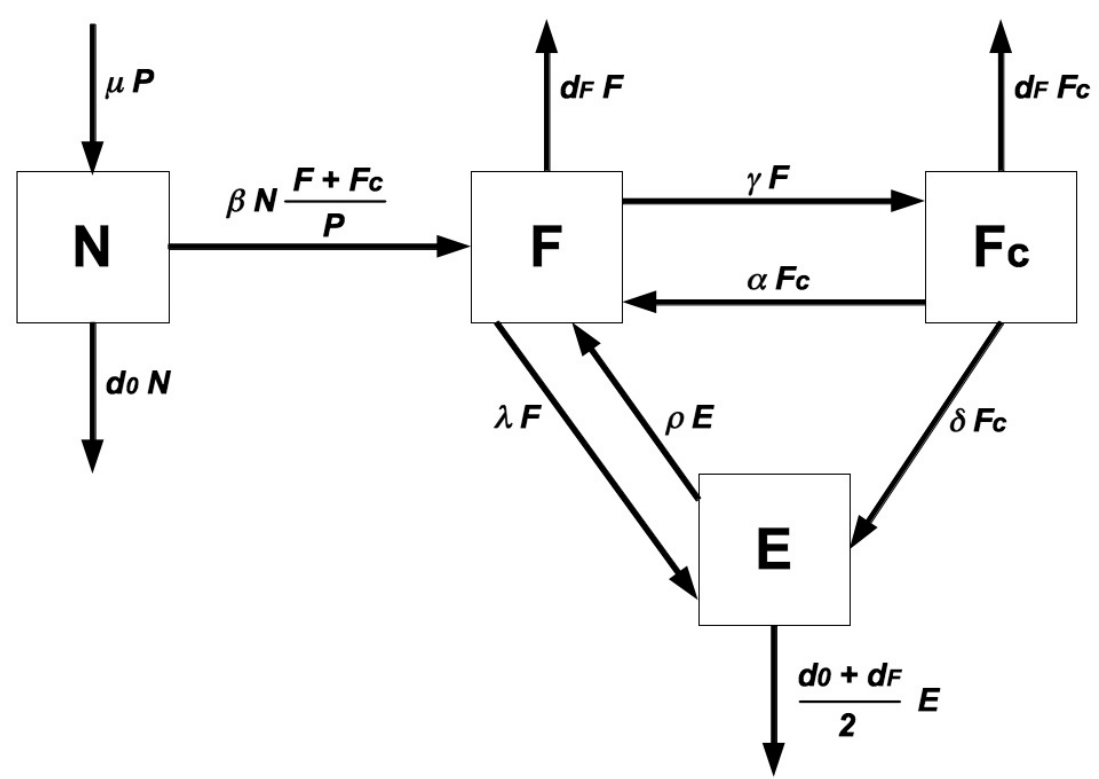

Figure 1: Flow diagram of the mathematical model for the dynamics of smoking prevalence in Spain. The boxes represent the subpopulations and the arrows represent the transitions between the subpopulations. Arrows are labeled by their corresponding model parameter.

The parameters of the model are defined as follows:

$\mu$ : birth rate in Spain.

$d 0$ : death rate in Spain.

$d F$ : increased death rate due to tobacco use.

$\beta$ : transmission rate due to social pressure to adopt smoking habit.

$\rho$ : rate at which an ex-smoker returns to smoking.

$\alpha$ : rate at which an excessive smoker becomes a normal smoker by reducing the number of cigarettes per day. 
$\gamma$ : rate at which a normal smoker becomes an excessive smoker by increasing the number of cigarettes per day.

$\lambda$ : rate at which normal smokers stop smoking.

$\delta$ : rate at which excessive smokers stop smoking.

The graph shown in Figure 1 represents a mathematical model described by a system of ordinary differential equations whose solution gives us the evolution of the four subpopulations. The details of the model building are explained in Appendix 1.

Since data in Table 1 are percentages of the total population we have to scale the equations into the same units as data, because one of our objectives is to fit data to the model. How we scale the equations is also shown in Appendix 1.

Hence, after scaling the equations we obtain the following system:

$$
\begin{aligned}
& d n d t=\mu-d o+\mu n+d o n 2+d F-\beta n f+f c+d o+d F 2 n e, \\
& d f d t=\beta n f+f c+\rho e+\alpha f c-\mu+\gamma+\lambda+d F f+d o n f+d F f f+f c+d o+d F 2 f e, \\
& d f c d t=\gamma f-\mu+\alpha+\delta+d F f c+d o n f c+d F f c f+f c+d o+d F 2 f c e, \\
& d e d t=\lambda f+\delta f c-\mu+\rho+d o+d F 2 e+d o n e+d F e f+f c+d o+d F 2 e 2,
\end{aligned}
$$

Where the new scaled variables are:

$n=N P, f=F P, f c=F c P, e=E P$,

while $\mathrm{P}$ denotes the total population.

This is the model we have used to predict tobacco use over next few years and to quantify the impact of legislation.

\section{Parameter estimations}

In order to obtain an accurate description of the evolution of the different subpopulations, first, we have to estimate the parameters. Each one of the parameters has been estimated as follows:

$\mu=0.01$ years $^{-1}$, birth rate in Spain. We have estimated this parameter taking into account the average Spanish rate between years 1993-2003. (Spanish Statistic Institute,2010).

$d 0=0.0087$ years $^{-1}$, is the average of the Spanish death rate between years 1993-2003 (Spanish Statistic Institute,2010).

$d F=0.0132$ years $^{-1}$, is the augmented death rate due to tobacco use (Montes et al.,2004). 
$\rho=0.0425$ years $^{-1}$, is the rate at which ex-smokers return to smoking habit (Agudo et al.,2004).

$\alpha=0.1244$ years $^{-1}$, rate at which an excessive smoker becomes a normal smoker by decreasing the number of cigarettes per day. We take the average rate for 1993-2003 (Ministry of Health and Social Policy,2006).

$\gamma=0.1175$ years $^{-1}$, is the rate at which normal smokers become excessive smokers by increasing the number of cigarettes per day. We take the average rate for 1993-2003 (Ministry of Health and Social Policy,2006).

$\lambda=0.0498$ years $^{-1}$, is the rate at which normal smokers stop smoking. We take the average rate for 1993-2003 (Ministry of Health and Social Policy,2006).

$\delta=0.0498$ years $^{-1}$, is the rate at which excessive smokers stop smoking. We take the average rate for 1993-2003 (Ministry of Health and Social Policy,2006).

Additionally, taking into account smoking prevalence in Spain (Table 1) we have estimated the parameter $\beta$ by fitting data with the model in the mean square sense. To find the value of $\beta$ that minimizes the mean square error, we used the Nelder-Mead algorithm (Nelder \& Mead,1964; Press et al.,1986) and we obtained $\beta=0.0381$. To see more details about the estimation of $\beta$ (Santonja et al.,2010).

Finally, with all the parameters estimated, we solved the system of differential equations using NDSolve[] command of Mathematica (Wolfram Research, 2010). Figure 2 shows the results of the model compared to real data from Table 1. 

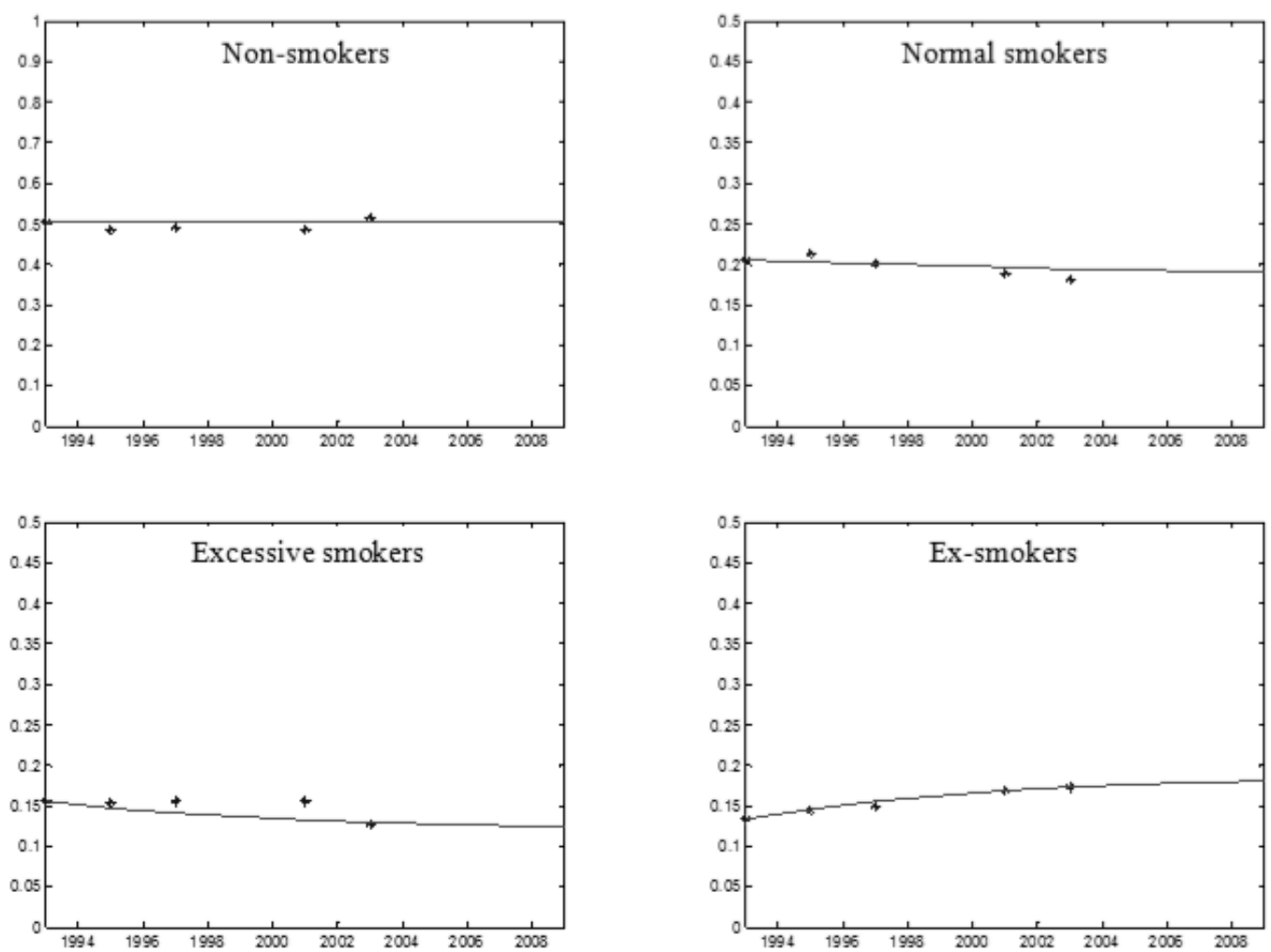

Figure 2: Numerical simulation of the fitted mathematical model where the points represent the real data from Table 1 and the lines are the solution of the model.

\section{RESULTS}

As we have said above, our objective is to quantify the impact of Spanish smoke-free law introduced in 2006. In order to achieve this, we have performed simulations with the mathematical model presented above for the period 1993-2009. We have shown that our model is valid to predict the evolution of the subpopulations without the effect of the law. Our fit has a mean square error of 0.0089 , or in percentage $0.89 \%$.

Our quantification analysis compares the model predictions for 2006 and 2009 (without the effect of the law) to real data (with the effect of the law) observed for 2006 (Ministry of Health and Social Policy,2006) and 2009 (Spanish Statistic Institute,2009). Real data for 2006 were collected at the end of 2006.

If the impact of the law was significant we would expect to see a change in the evolution of subpopulations before and after the law. To see whether that change happened, we extrapolated the model until 2009 to see what would had happened without the law and then we compared it with the real situation given by the data for 2006 and 2009.

Table 2 shows the comparison between predicted and real data for 2006 and 2009.

$<<$ Insert Table 2>> 
The first column shows the four subpopulations included in the model and the total proportion of smokers. This last group is included because in (Spanish Statistic Institute,2009) the group of smokers is not subdivided into normal and excessive smokers.

The second and third columns show real and predicted values respectively. The fourth column calculates the difference between real and predicted values, or in other words the deviation with respect to the model. And the fourth column shows whether these deviations are significant or not.

As we can see in Figure 2 our model does not fit real data exactly. There is an average deviation of 0.0089 per point. Therefore we have to compare the deviations in Table 2, with the average deviation in order to distinguish whether deviations for 2006 and 2009 are attributable to the model or to the impact of the law.

For example, a value of 3 for the quotient Df/MSE means that the deviation between predicted and real data for that observation is three times larger than the normal model deviation and this suggest that there is an effect only attributable to the impact of the smoking-free law.

According to this, we consider significant the deviations with a value of Df/MSE around 2 or more in absolute value.

Therefore, taking into account Df/MSE ratios, we observe in Table 2 the following facts:

1. Normal smoker + excessive smoker population decreased in 2006. Real data (0.2950) is smaller than the predicted value (0.3186) and this difference is significant (-2.652). However, we also observe that this trend disappears in 2009. In this case, real data is around the predicted value (without the effect of the law). The difference is 0.0001 , much smaller than the mean square error.

2. The excessive smoker population decreased in 2006. The observed value (0.1094) is smaller than the predicted value (0.1264) and the difference is significant (Df/MSE=-1.910).

3. The percentage of ex-smokers increased in 2006. In this case, real data (0.2054) is greater than the predicted value (0.1773) and this difference is very significant $(\mathrm{Df} / \mathrm{MSE}=3.146)$. Unfortunately, this difference did not grow over time. We note that real data for $2009(0.2017)$ is again greater than the predicted value $(0.1805)$ and the difference is still significant (Df/MSE=2.382), but it is smaller than before.

4. Finally, we see a significant difference in non-smokers in 2009 . The observed value $(0.4835)$ is smaller than the predicted value (0.5049), and the difference is significant (Df/MSE=-2.404). It is remarkable because this did not happen in 2006 when the difference was not relevant.

Summarising, we can consider the effect of the law in the first moment as consisting of a significant decrease in the number of smokers (both normal and excessive), especially in excessive smokers, and a corresponding increase in ex-smokers. The differences between real data and predicted values are quite significant. We can say that the global effect of the law in 2006 was around $2 \%$ of the total population between 16 and 65 years old. We see this $2 \%$ difference between predicted and real number of smokers ( $0.2950 \mathrm{vs.} 0.3186)$ and between predicted and real number of ex-smokers ( 0.2053 vs. 0.1773$)$

However, this trend stopped in 2009. In the survey for 2009, the difference between the predicted and the real number of ex-smokers is maintained around $2 \%(0.2017 \mathrm{vs} .0 .1805)$, but the number of smokers coincides with the predicted value. It is also very significant that the proportion of ex-smokers is the same in 2006 as in 2009. 
Therefore, we can say that this effect of the law disappeared in 2009. These results are in accordance with the results presented in other studies (Ministry of Health and Social Policy,2009; Galan\&Lopez,2009).

\section{CONCLUSIONS}

A mathematical model for smoking behavior is presented and studied. First, identification of nonsmokers, normal smokers, excessive smokers and ex-smokers are performed. Then, population is divided into four groups according to smoking habits and a compartmental model is constructed. Future short term addicted population is computed and the impact of the Spanish smoke-free legislation is analysed.

According to the results shown above, we can conclude the following:

1. When the law came into force (2006) many people decided to give up smoking. Then, the number of smokers decreased by $2.3 \%$ and, correspondingly, the number of ex-smokers increased also by $2.3 \%$.

2. Three years later, in 2009 , most of the people who had succeeded in giving up smoking did not suffer relapse. This means that about 770,000 people stopped smoking due to the law (that is, the $2 \%$ of the total population between 16 and 65 years old).

3. However, three years later, the law had no effect on new smokers, as we see in the decreasing number of non smokers and the increasing number of smokers in 2009. To reduce the increasing of new smokers, probably the law should be backed up by other more specific policies.

In this modeling approach, we show how mathematical models can be a useful tool to understand smoking habit spread. Using this type of models, policy makers can provide insight into this problem and quantify the impact of public health strategies.

\section{ACKNOWLEDGMENTS}

\section{REFERENCES}

Agudo, A., et al. (2004). Changes in smoking habits in adults: results from a prospective study in Spain. Annals of Epidemiology,14,235-243.

Banegas, J.R., et al. (2005) La mortalidad atribuible al tabaquismo comienza a disminuir en España (Deaths attributable to smoking is decreasing in Spain). Medicina Clínica (Barc), 124,769-771.

Christakis, N.A., Fowler, J.H. (2008). The collective dynamics of smoking in a large social network. The New England Journal of Medicine,358,2249-2258.

Christakis, N.A., Fowler, J.H. (2009).Connected: the surprising power of our social networks and how they shape our lives. Brown and Company. Hachette Book Group.

Erazo, M., et al. (2010). Secondhand tobacco smoke in bars and restaurants in Santiago, Chile: evaluation of partial smoking ban legislation in public places. TobControl. tc.2009.035402. Published Online First: 25 August 2010. doi:10.1136/tc.2009.035402 
Galan, I., Lopez, M.J. (2009). Three years with Tobacco-control law: cleaner air but not clean enough. Gaceta Sanitaria,23,87-90.

Lock, K., et al. (2010). Evaluating social and behavioral impacts of English smoke-free legislation in different ethnic and age groups: implications for reducing smoking-related health inequalities. Tobacco Control,19,391-397.

Martcheva, M., Castillo-Chávez, C. (2003). Diseases with cronic stage in a population with varying size. Mathematical Biosciences, 182,1-25.

Mena-Lorca, J., Hethcote, H.W. (1992). Dynamic models of infectious diseases as regulators of population sizes. Journal of Mathematical Biology,30,693-716.

Ministry of Health and Social Policy. (2005). Law 28/2005 Health policies against smoking and the regulation of selling, consumption and publicity of tobacco products. Spanish Official Bulletin,309,42241-42249.

Ministry of Health and Social Policy, (2006). National Health Survey for Spain: 1987-2006.

Retrieved 21st November 2010 from http://www.msc.es/estadEstudios/ estadisticas/encuestaNacional/home.htm

Ministry of Health and Social Policy. (2009). Evaluación del impacto de la ley de medidas sanitarias frente al tabaquismo (Evaluation of the impact of the smoke-free law). Retrieved 15 th May 2010 from http://www.msps.es/ciudadanos/proteccionSalud/tabaco/ docs/monografiaEvaluacionLey282005.pdf.

Montes, A., Perez, M., Gestal, J.J. (2004). Impacto del tabaquismo sobre la mortalidad en España (Smoking effect on death rate in Spain). Revista Adicciones, 16,75-82.

Murray, J.D. (2002). Mathematical Biology. Springer.

Nelder, J.A., Mead, R. (1964). A simplex method for function minimization. Computer Journal, 7, 308-313.

Press, W.H., et al. (1986). Numerical recipes: the art of scientific computing. Cambridge University Press.

Sánchez, E., et al. (2010). Predicting cocaine consumption in Spain: a mathematical modeling approach. Drugs: Education, Prevention and Policy. Published Online First: 5 August 2010. doi:abs/10.3109/09687630903443299.

Santonja, F.J., et al. (2010). Alcohol consumption in Spain and its economic cost: a mathematical modeling approach. Mathematical and Computer Modelling,52,999-1003.

Spanish Statistic Institute.(2009). European Health Survey 2009. Retrieved 21st November 2010 from http://www.ine.es/inebmenu/mnu salud.htm.

Spanish Statistic Institute, (2010). Population Census. Retrieved http://www.ine.es .

Wolfram Research.(2010). Software for scientific computing. Retrieved 21st November 2010 from http://www.wolfram.com.

\section{APPENDIX 1}

The model shown in Figure 1 is described by a system of ordinary differential equations whose solution gives us the evolution over time for the four subpopulations. In order to build the model, we need to make some assumptions: 
-For this model, transition time-constant parameters are more suitable since we want to model the evolution of smoking habit over a short time.

-The subpopulation sizes and their behavior over time will determine the dynamic evolution of the smoking habit.

-Complete homogeneous mixing in which every person in a subpopulation can interact with people from all other groups (Murray,2002).

-The transitions between the subpopulations $F t, F c(t)$ and $E(t)$ are governed by terms proportional to the sizes of these subpopulations.

-However, the transition from $N(t)$ to $F t$ occurs through the transmission of the smoking habit from smokers to non-smokers, depending on the encounters among them. This transit is modeled by the term $\beta N(t)[F t+F c t] / P(t)$.

-We are assuming that when ex-smokers relapse back to smoking they do it gradually, so they enter in the subpopulation Ft. Hence, we do not consider the migration between $E(t)$ and $F c(t)$ in our model.

The detailed system of equations is the following ( $\mathrm{t}$, time in years):

$$
\begin{aligned}
& N^{\prime}(t)=\mu P(t)-d o N(t)-\beta N(t)[F t+F c t] P(t) \\
& F^{\prime}(t)=\beta N t F t+F c t P t+\rho E(t)+\alpha F c(t)-\gamma+\lambda+d F F(t) \\
& F c^{\prime}(t)=\gamma F(t)-\alpha+\delta+d F F c(t) \\
& E^{\prime}(t)=\lambda F(t)+\delta F c(t)-\rho+d o+d F 2 E(t) \\
& P t=N t+F t+F c t+E(t)
\end{aligned}
$$

Data obtained in Table 1 are related to the percentages of population, but the equations of the model shown above are related to the number of individuals. This leads us to transform (by scaling) the model into the same units as data, in order to compare predictions with data.

Hence, following ideas developed in (Martcheva \& Castillo-Chavez,2003; Mena-Lorca \& Hethcote, 1992) about how to scale models where the population is varying in size, we obtain the equations of the model as follows. Adding equations of the model above one gets:

$$
P^{\prime} t=\mu P t-d 0 N t-d F F t+F c t-d 0+d F 2 E t \text {. }
$$

Dividing both members by $P t$ we have that: 
$P^{\prime}(t) P(t)=\mu-d 0 N t P t-d F F(t) P(t)+F c t P t-d 0+d F 2 E(t) P(t)$.

(7)

If we define the following rates (depending on time)

$n=N P, f=F P, f c=F c P, e=E P$

equation (7) can be transformed into

$P^{\prime} P=\mu-d 0 n-d F f+f c-d 0+d F 2 e$.

Then, we compute the derivatives of $n, f, f c$ and $e$ defined in (8) using (1-5), (8) and (9). We obtain:

$d n d t=\mu-d o+\mu n+d o n 2+d F-\beta n f+f c+d o+d F 2 n e$

$d f d t=\beta n f+f c+\rho e+\alpha f c-\mu+\gamma+\lambda+d F f+d o n f+d F f f+f c+d o+d F 2 f e$

$d f c d t=\gamma f-\mu+\alpha+\delta+d F f c+d o n f c+d F f c f+f c+d o+d F 2 f c e$

$d e d t=\lambda f+\delta f c-\mu+\rho+d o+d F 2 e+d o n e+d F e f+f c+d o+d F 2 e 2$

The solution of this scaled model allows us to obtain the prediction that the model gives for the evolution of the subpopulations of non-smokers, smokers (normal and excessive) and ex-smokers. Since they are in the same units as data, we can compare them directly. 


\begin{tabular}{|c|c|c|c|c|}
\hline & $\mathrm{N}$ & $\mathrm{F}$ & $\mathrm{F}_{\mathrm{c}}$ & $\mathrm{E}$ \\
\hline 1993 & $50.45 \%$ & $20.59 \%$ & $15.59 \%$ & $13.37 \%$ \\
\hline 1995 & $48.56 \%$ & $21.46 \%$ & $15.45 \%$ & $14.53 \%$ \\
\hline 1997 & $49.22 \%$ & $20.17 \%$ & $15.61 \%$ & $15.00 \%$ \\
\hline 2001 & $48.69 \%$ & $18.98 \%$ & $15.50 \%$ & $16.83 \%$ \\
\hline 2003 & $51.69 \%$ & $18.20 \%$ & $12.77 \%$ & $17.34 \%$ \\
\hline
\end{tabular}

Table 1. Evolution of the proportion of non-smokers $(\mathrm{N})$, normal smokers $(\mathrm{F})$, excessive smokers $\left(\mathrm{F}_{\mathrm{c}}\right)$ and exsmokers (E) for different years (Ministry of Health and Social Policy,2006). 


\begin{tabular}{|l|l|l|l|l|}
\hline 2006 & Real data & Predicted value & Difference (Df) & Df/MSE \\
\hline Non-smokers & 0.4997 & 0.5041 & -0.0044 & -0.494 \\
\hline Normal smokers & 0.1856 & 0.1902 & -0.0066 & -0.742 \\
\hline $\begin{array}{l}\text { Excessive } \\
\text { smokers }\end{array}$ & 0.1094 & 0.1264 & -0.0170 & -1.910 \\
\hline Ex-smokers & 0.2053 & 0.1773 & 0.0280 & 3.146 \\
\hline All smokers & 0.2950 & 0.3186 & -0.0236 & -2.652 \\
\hline & & & & \\
\hline 2009 & Real data & Predicted value & Difference (Df) & Df/MSE \\
\hline Non-smokers & 0.4835 & 0.5049 & -0.0214 & -2.404 \\
\hline Normal smokers & NA & 0.1906 & NA & NA \\
\hline $\begin{array}{l}\text { Excessive } \\
\text { smokers }\end{array}$ & NA & 0.1240 & NA & NA \\
\hline Ex-smokers & 0.2017 & 0.1805 & 0.0212 & 2.382 \\
\hline All smokers & 0.3147 & 0.3146 & 0.0001 & 0.011 \\
\hline
\end{tabular}

Table 2: Real data vs. Predicted values for 2006 and 2009. MSE is the mean square error of the model, presented above. NA: non-available data in (Spanish Statistic Institute,2009). 